\title{
RESEARCH BRIEF \\ Flavored Cannabis Product Use Among Adolescents in California
}

\author{
Miranda Werts, $\mathrm{BA}^{1}$; Janelle Urata, RDH, $\mathrm{MS}^{1}$; Shannon Lea Watkins, $\mathrm{PhD}^{2}$; \\ Benjamin W. Chaffee, DDS, MPH, PhD $^{1}$
}

\begin{abstract}
Accessible Version: www.cdc.gov/pcd/issues/2021/21_0026.htm
Suggested citation for this article: Werts M, Urata J, Watkins SL, Chaffee BW. Flavored Cannabis Product Use Among Adolescents in California. Prev Chronic Dis 2021;18:210026. DOI: https:// doi.org/10.5888/pcd18.210026.
\end{abstract}

\section{PEER REVIEWED}

\section{Summary}

What is already known on this topic?

Cannabis use among youth is increasing. Flavors in tobacco products are associated with youth appeal and experimentation.

\section{What is added by this report?}

In this sample of California adolescents, approximately half of those who recently used smoked cannabis and the majority who used aerosolized ("vaped") cannabis reported using a flavored product, most often fruit or dessert, in the past month.

What are the implications for public health practice?

Within an expanding legal cannabis marketplace, this report highlights a potential role for restrictions on marketing cannabis flavors and flavored additives, including flavored cigar and vaporizer products often used to consume cannabis, to discourage cannabis use among adolescents.

\section{Abstract}

Given the well-documented role of flavors in encouraging tobacco use among adolescents and diversity of the cannabis market, we describe flavored cannabis product use, both smoked and aerosolized ("vaped"), among a sample of adolescents. We surveyed 1,423 students in 8 Northern and Central California public high schools (2019-2020) to record flavored tobacco and cannabis use. Among past 30-day cannabis users, use of flavored cannabis, most often fruit-flavored, was common for smoked (48.1\%) and vaped $(58.0 \%)$ products. Given that youth-appealing flavors may contribute to underage cannabis use, emerging cannabis control policies should consider lessons from tobacco control to prevent youth cannabis use.

\section{Objective}

Cannabis (marijuana) is frequently used by US high school students, with almost $36 \%$ of 12 th-grade students reporting past-year use (1). Cannabis products, including oil vaporizers, cannabis flower, and blunt wraps, are advertised with flavor, taste, and food descriptors (eg, "sweet," "fruit," "lemon haze") (2). Among adolescent tobacco users, use of flavored products, particularly for electronic cigarettes (e-cigarettes), is common (3) and reported as a motivation for tobacco use (4). Little work has documented patterns of flavored cannabis product use (5), particularly among youth. Here, we describe flavor-related behaviors among adolescent tobacco and cannabis users to help inform potential cannabis control policy.

\section{Methods}

An in-person, school-based survey was administered to grade 9 and 10 students $(\mathrm{N}=1,423)$ in 8 public high schools in Northern and Central California in 2019-2020, as described elsewhere (6). Schools were in municipalities with fewer than 50,000 residents and in counties of population density less than 1,000 people per square mile. The survey assessed both past 30-day and ever use of tobacco and cannabis, perceptions of tobacco products, socioenvironmental variables, and health conditions. Participating students received a $\$ 10$ gift card to an online retailer and each school received $\$ 300$. Parents provided written consent and students assented. The institutional review board at the University of California, San Francisco, approved all study procedures.

Our cross-sectional analysis examined baseline data collected from March 2019 to February 2020. Ever and past 30-day use prevalence of cigarettes, cigars, e-cigarettes, moist snuff, and cannabis were calculated for the entire sample based on separate survey items for each product that included brief product descriptions and photographs. Students who reported past 30-day cannabis use were asked to report how they consumed cannabis from a list of methods, categorized as smoked (joints, blunts, spliffs, pipes, bong, or moke), vaped (vaporized or dabbed flower, bud, 
oil, wax, extract, liquid, or concentrate), or other (eg, edible or tincture).

Separately for each tobacco product and for smoked and vaped cannabis routes of administration, past 30-day users were asked which flavors they had used in the past 30 days (the options were not mutually exclusive), later grouped as "flavored," "not flavored or tobacco flavor only," and "don't know." Among cannabis past 30-day users (smoked and/or vaped), we also calculated the unweighted frequency of reporting each specific flavor (eg, fruit, alcoholic drink). This descriptive analysis does not include hypothesis tests and was conducted using Stata 16.0 (StataCorp LLC).

\section{Results}

Approximately half of the sample identified as female (53.4\%), as Hispanic or Latino (53.1\%), and/or as qualified for free or reduced-price school lunches (54.2\%). More than one-third of adolescents had ever used cannabis $(37.7 \%)$ or tobacco $(44.6 \%$, any tobacco product, including e-cigarettes). Prevalence of past 30-day use was $21.0 \%$ for cannabis (any form) and $23.2 \%$ for tobacco (any product, including e-cigarettes) (Table 1).

Flavored product use was common for tobacco and cannabis. Most past 30-day users of moist snuff (68.4\%), cigars $(80.6 \%)$, and ecigarettes $(75.8 \%)$ used flavored products, and $43.8 \%$ of past 30 day cigarette users reported smoking mint or menthol cigarettes (Table 1). Among smoked cannabis users $(n=160), 48.1 \%$ reported using a flavored smoked cannabis product, and 58.0\% among vaped cannabis users $(\mathrm{n}=139)$ reported using a flavored aerosolized cannabis product (Table 1).

Among responses indicating a flavor, fruit was the most common category for both smoked (30.4\%) and vaped (39.1\%) cannabis (Table 2). Candy, dessert, or other sweet flavors was the next most common flavor category, whereas use of other flavors such as alcoholic drink, mint, and menthol was infrequently reported (Table 2).

\section{Discussion}

These results show that a substantial proportion of adolescent cannabis users are choosing flavored cannabis products, including both combustible and aerosolized products. This finding illuminates a potential health concern, as adolescents associate flavors in tobacco products with less perceived harm and have greater interest in experimentation with flavored tobacco compared with unflavored tobacco products $(6,7)$. Flavored cannabis might have similar effects. Many US states permit or have decriminalized medical and/or recreational cannabis use (8), corresponding with rising levels of cannabis use among youth and adults (9). As the commercial cannabis industry offers an expanding array of products promoted as flavored, potential restrictions on flavored cannabis may prove an important component of limiting youth appeal.

What is considered flavored cannabis may reflect a variety of use patterns. Smoked cannabis may be consumed by using flavored rolling papers, as a blunt within a flavored tobacco cigar $(10,11)$, or with cannabis flower that was marketed with flavor descriptors (2). Questionnaire items did not differentiate between flavors as additives, wrappers, or taste characteristics of cannabis strains; future work should consider these differences. Using flavored cigar products for cannabis consumption is particularly popular among cannabis users $(5,10)$, suggesting that potential coordination of tobacco and cannabis flavor restriction policies may beneficially reduce both tobacco and cannabis use among youths.

This study had limitations. Results from this sample of California schools in smaller towns may not generalize to other geographic contexts; e-cigarette and cannabis use were higher than from earlier statewide estimates (12). The sample size precluded precise estimates of flavor use prevalence. As markets co-evolve, future work should examine associations between flavored cannabis and flavored tobacco use, including their co-administration and use patterns longitudinally.

The observed levels of flavored cannabis and flavored tobacco use in this study underscore the relevance of flavors in both tobacco and cannabis control policy. Restrictions that prohibit sales of any characterizing flavors, such as recent local and state restrictions on the sale of flavored tobacco products (eg, New York City [13] and San Francisco [14]), could help address rising adolescent interest in new tobacco products and cannabis use.

\section{Acknowledgments}

The first 2 authors contributed equally to this article. Thanks to Elizabeth Couch of the University of California, San Francisco, and David Cash and Bonnie Halpern-Felsher of Stanford University for supporting project design, administration, and data collection. This work was supported by the National Institutes of Health (grant number U54HL147127). The funder had no role in carrying out the research nor in publication. Content is the responsibility of the authors and does not necessarily reflect the official views of the funding agency. No copyrighted materials (figures, images, photos, surveys, or tools) were used in this publication.

\footnotetext{
The opinions expressed by authors contributing to this journal do not necessarily reflect the opinions of the U.S. Department of Health and Human Services, the Public Health Service, the Centers for Disease Control and Prevention, or the authors' affiliated institutions.
} 


\section{Author Information}

Corresponding Author: Benjamin W. Chaffee, University of California, San Francisco, 3333 California St, Ste 495, San Francisco, CA 94118. Telephone: 415-476-9226. Email: benjamin.chaffee@ucsf.edu.

Author Affiliations: ${ }^{1}$ University of California, San Francisco, California. ${ }^{2}$ University of Iowa, Iowa City, Iowa.

\section{References}

1. National Institute on Drug Abuse. Monitoring the Future 2019 survey results: overall findings. 2019. https:// www.drugabuse.gov/drug-topics/trends-statistics/infographics/ monitoring-future-2019-survey-results-overall-findings. Accessed September 22, 2020.

2. Luc MH, Tsang SW, Thrul J, Kennedy RD, Moran MB. Content analysis of online product descriptions from cannabis retailers in six US states. Int J Drug Policy 2020;75:102593.

3. Cullen KA, Liu ST, Bernat JK, Slavit WI, Tynan MA, King $\mathrm{BA}$, et al. Flavored tobacco product use among middle and high school students - United States, 2014-2018. MMWR Morb Mortal Wkly Rep 2019;68(39):839-44.

4. Wang TW, Gentzke AS, Creamer MR, Cullen KA, HolderHayes E, Sawdey MD, et al. Tobacco product use and associated factors among middle and high school students United States, 2019. MMWR Surveill Summ 2019; 68(12):1-22.

5. Rosenberry ZR, Schauer GL, Kim H, Peters EN. The use of flavored cigars to smoke marijuana in a sample of US adults co-using cigarettes and marijuana. Tob Regul Sci 2017;3(2, Suppl 1):94-100.

6. Chaffee BW, Couch ET, Urata J, Cash D, Werts M, HalpernFelsher B. Electronic cigarette and moist snuff product characteristics independently associated with youth tobacco product perceptions. Tob Induc Dis 2020;18(August):71.

7. Kowitt SD, Meernik C, Baker HM, Osman A, Huang L-L, Goldstein AO. Perceptions and experiences with flavored nonmenthol tobacco products: a systematic review of qualitative studies. Int J Environ Res Public Health 2017;14(4):338.

8. DISA Global Solutions. Map of marijuana legality by state. https://disa.com/map-of-marijuana-legality-by-state. Published 2020. Updated November 4, 2020. Accessed December 3, 2020.
9. Cerdá M, Mauro C, Hamilton A, Levy NS, Santaella-Tenorio $\mathrm{J}$, Hasin D, et al. Association between recreational marijuana legalization in the United States and changes in marijuana use and cannabis use disorder from 2008 to 2016. JAMA Psychiatry 2020;77(2):165-71.

10. Giovenco DP, Miller Lo EJ, Lewis MJ, Delnevo CD. "They're pretty much made for blunts": product features that facilitate marijuana use among young adult cigarillo users in the United States. Nicotine Tob Res 2017;19(11):1359-64.

11. Knapp AA, Lee DC, Borodovsky JT, Auty SG, Gabrielli J, Budney AJ. Emerging trends in cannabis administration among adolescent cannabis users. J Adolesc Health 2019; 64(4):487-93.

12. Zhu S-H, Zhang Y-L, Braden K, Cole A, Gamst A, Wolfson T, et al.Results of the Statewide 2017-18 California Student Tobacco Survey. San Diego (CA): Center for Research and Intervention in Tobacco Control (CRITC), University of California, San Diego; 2019.

13. NYC Health. Flavored tobacco and vaping products. https:// www1.nyc.gov/site/doh/health/health-topics/flavored-tobaccoand-vaping-products.page. Accessed May 4, 2021.

14. San Francisco Department of Health. Environmental Health. Flavored tobacco. https://www.sfdph.org/dph/EH/Tobacco/ flavoredtobacco.asp. Accessed May 4, 2021.

The opinions expressed by authors contributing to this journal do not necessarily reflect the opinions of the U.S. Department of Health and Human Services, the Public Health Service, the Centers for Disease Control and Prevention, or the authors' affiliated institutions. 


\section{Tables}

Table 1. Flavored Product Use Among Past 30-Day Users, by Product ( $\mathrm{N}=1,423)$, California High School Students, 2019-2020

\begin{tabular}{|c|c|c|c|c|c|c|}
\hline Product & Cigarettes & Moist Snuff & Cigars & E-Cigarettes & Smoked ${ }^{b}$ Cannabis & Vaped $^{\mathrm{C}}$ Cannabis \\
\hline $\begin{array}{l}\text { Past 30-day product use, } \\
\text { among total sample }\end{array}$ & $32(2.2)$ & $20(1.4)$ & $36(2.5)$ & $302(21.2)$ & $160(11.3)$ & $139(9.8)$ \\
\hline \multicolumn{7}{|c|}{ Flavored use, among past 30-day users ${ }^{d}$} \\
\hline Flavored $^{\mathrm{e}}$ & $14(43.8)$ & $13(68.4)$ & $29(80.6)$ & $225(75.8)$ & $76(48.1)$ & $80(58.0)$ \\
\hline No flavor or tobacco flavor ${ }^{f}$ & $15(46.9)$ & $6(31.6)$ & $7(19.4)$ & $27(9.1)$ & $39(24.7)$ & $24(17.4)$ \\
\hline Don't know & $3(9.4)$ & $0(0.0)$ & $0(0.0)$ & $45(15.2)$ & $43(27.2)$ & $34(24.6)$ \\
\hline Missing data ${ }^{\mathrm{g}}$ & 0 & 1 & 0 & 5 & 2 & 1 \\
\hline
\end{tabular}

a Values are number (unweighted percentage).

${ }^{\mathrm{b}}$ Includes joints, blunts, spliffs, pipes, bong, or moke (cannabis and tobacco waterpipe).

${ }^{c}$ Includes vaped or dabbed flower, bud, oil, wax, extract, liquid, or concentrate.

${ }^{d}$ Percentages may not add to $100 \%$ because of rounding.

e Includes only mint or menthol for cigarettes; users of other products selected $\geq 1$ option from a list of flavor categories (eg, fruit, mint, dessert, other [Table 2]).

${ }^{\mathrm{f}}$ Used only nonflavored or tobacco-flavored products in the past 30 days.

${ }^{g}$ Missing values excluded from denominator for calculating percentages. 
Table 2. Types of Flavors Used Among Past 30-Day Cannabis Users, California High School Students, 2019-2020

\begin{tabular}{|c|c|}
\hline Flavor & No. (Unweighted Percentage) ${ }^{a}$ \\
\hline \multicolumn{2}{|l|}{ Smoked cannabis $(n=160)$} \\
\hline No flavor ${ }^{b}$ & $56(35.4)$ \\
\hline Fruit & $48(30.4)$ \\
\hline Candy, dessert, or sweet & $38(24.1)$ \\
\hline Alcoholic drink & $9(5.7)$ \\
\hline Spice or cinnamon & $8(5.1)$ \\
\hline Mint (not menthol) & $6(3.8)$ \\
\hline Menthol (cool or frost) & $4(2.5)$ \\
\hline Nonalcoholic drink & $0(0.0)$ \\
\hline Other flavor & $8(5.1)$ \\
\hline Don't know ${ }^{c}$ & $48(30.4)$ \\
\hline Missing data ${ }^{d}$ & 2 \\
\hline \multicolumn{2}{|l|}{ Vaped cannabis $(n=139)$} \\
\hline Fruit & $54(39.1)$ \\
\hline No flavor ${ }^{b}$ & $43(31.2)$ \\
\hline Candy, dessert, or sweet & $38(27.5)$ \\
\hline Alcoholic drink & $8(5.8)$ \\
\hline Mint (not menthol) & $7(5.1)$ \\
\hline Menthol (cool or frost) & $5(3.6)$ \\
\hline Spice or cinnamon & $5(3.6)$ \\
\hline Nonalcoholic drink & $2(1.4)$ \\
\hline Other flavor & $2(1.4)$ \\
\hline Don't know ${ }^{\mathrm{C}}$ & $41(29.7)$ \\
\hline Missing data ${ }^{d}$ & 1 \\
\hline
\end{tabular}

a Not mutually exclusive: respondents could select $\geq 1$ flavor option, including "don't know" and "no flavor."

b Not mutually exclusive: includes those who selected "no flavor" even if another flavor was also selected.

'Not mutually exclusive: includes those who selected "don't know" even if another flavor was also selected.

${ }^{\mathrm{d}}$ Missing values excluded from denominator for calculating percentages. 